(C) 1999 International Press

Adv. Theor. Math. Phys. 3 (1999) 343-361

\title{
Summing Up Instantons in Three-Dimensional Yang-Mills Theories
}

\author{
Sonia Paban ${ }^{a}$, Savdeep Sethi ${ }^{b}$, Mark Stern $^{c}$ \\ ${ }^{a}$ Theory Group, \\ Department of Physics, \\ University of Texas, \\ Austin, TX 78712, USA \\ paban@zippy .ph.utexas.edu \\ ${ }^{b}$ School of Natural Sciences, \\ Institute for Advanced Study, \\ Princeton, NJ 08540, USA \\ sethi@sns.ias.edu \\ ${ }^{c}$ Department of Mathematics, \\ Duke University, \\ Durham, NC 27706, USA \\ stern@math.duke.edu
}

\begin{abstract}
We show that the four derivative terms in the effective action of three-dimensional N=8 Yang-Mills theory are determined by supersymmetry. These terms receive both perturbative and non-perturbative corrections. Using our technique for constraining the effective action, we are able to determine the exact form of the eight fermion terms in the supersymmetric completion of the $F^{4}$ term, including all instanton corrections. As a consequence, we argue that the integral of the Euler density over $k$ monopole moduli space in $S U(2)$ Yang-Mills is determined by our non-renormalization theorem for all values of $k$.
\end{abstract}

e-print archive: http://xxx.lanl.gov/abs/hep-th/9808119 


\section{Introduction}

In three dimensions unlike other dimensions, the effective action of YangMills theory with sixteen supersymmetries is expected to receive non-perturbative corrections at low orders in a derivative expansion. Let us recall that for $U(N)$ gauge theory, the effective action at generic points on the Coulomb branch is described by a sigma model with $7 N$ scalars $\phi^{i}$ where $i=1, \ldots, 7$. There are also $N$ additional scalars obtained by dualizing the $N$ abelian gauge fields. We will only consider the effective action which is local in terms of the dual scalars, rather than local in terms of the gauge-fields. The coupling constant in the theory, $g^{2}$, has mass dimension one. Therefore to study the infra-red theory, we take the coupling $g^{2} \rightarrow \infty$. The metric on the moduli space is flat and if we take canonical kinetic terms for all fields including the dual scalars $\sigma$,

$$
S=\frac{1}{g^{2}} \int d^{3} x \frac{1}{2} \partial_{\mu} \sigma \partial^{\mu} \sigma+\sum_{i} \frac{1}{2} \partial_{\mu} \phi^{i} \partial^{\mu} \phi^{i}
$$

then the dual scalars are periodic with a period proportional to $g^{2}$. The moduli space is then $S^{N}\left(\mathbb{R}^{7} \times S^{1}\right)$. At the origin of the moduli space, the theory flows to an interacting $\operatorname{Spin}(8)$ invariant fixed point in the infra-red $[1,2,3]$. The $\operatorname{Spin}(8)$ invariance is crucial if matrix theory $[4]$ is to correctly describe the light-cone quantized type IIB string. For the most part, we will consider the $S U(2)$ theory.

While the metric on the moduli space of the theory is flat, the higher derivative terms in the effective action must receive non-perturbative corrections, or the effective action will fail to be $\operatorname{Spin}(8)$ invariant in the strong coupling limit. The one instanton contribution to the $F^{4}$ terms was first computed in [5]. Subsequently, the computation was extended to multi-instanton effects in [6]. An independent argument for the existence of instanton contributions to the $F^{4}$ terms was presented in [7]. The importance of the instanton corrections for the matrix description of the type IIB string was discussed in [8]. The aim of this paper is to show that the same technique used in $[9,10]$ easily extends to this case as briefly mentioned in [9]. We will be able to completely determine the form of the four derivative terms in the effective action, including all perturbative and non-perturbative effects.

For the four derivative terms, the various contributions to the effective action take the form of a one-loop contribution together with perturbative corrections around multi-instanton configurations. It is quite remarkable that the instanton corrections are completely captured by the solution of a family of first order differential equations. Relations of a similar flavor have 
appeared in numerous places in both string theory and field theory.

By matching the leading instanton contributions to semi-classical computations [6], we are able to extract the integral of the Euler density over $k$ monopole moduli space for all $k$. This is completely analogous to the way that the bulk term for the $L^{2}$ index for $k$ D0-branes $[11,12]$ was extracted from the coefficient of the $\mathcal{R}^{4}$ term in type IIB supergravity [13]. The coefficient of the $\mathcal{R}^{4}$ term is also determined by supersymmetry [14]. In that case, a proof of the conjectured relation was provided by [15]. In this case involving monopoles, the predicted data is more geometrical since it involves the behavior of the metric on $k$ monopole moduli space. ${ }^{1}$ This fascinating relation between the properties of various soliton configurations and supersymmetry constraints cries out for a deeper explanation. Lastly, we should stress that the same techniques used for $F^{4}$ can be used to determine the $F^{6}$ terms in three dimensions as in [10].

\section{Determining the Four Derivative Terms in Three Dimensions}

\subsection{An equation for the eight fermion terms}

For simplicity, let us use $\phi^{8}$ to denote the dual scalar $\sigma$. For any finite coupling, the symmetry group in three dimensions is $S O(2,1) \times \operatorname{Spin}(7)$, where $S O(2,1) \sim S L(2, \mathbb{R})$ is the Lorentz group. The scalars $\phi^{i}$ where $i=1, \ldots, 7$ transform in the $(\mathbf{1}, \mathbf{7})$ of $S O(2,1) \times \operatorname{Spin}(7)$. There are also fermions $\psi_{\alpha a}$ which transform in the $(\mathbf{2}, \mathbf{8})$ of the symmetry group, where $\alpha=1,2$ and $a=1, \ldots, 8$.

While the manifest symmetry group of the Lagrangian is only $\operatorname{Spin}(7)$, the lowest order supersymmetry transformations actually exhibit the full infra-red $\operatorname{Spin}(8)$ invariance. We will shift between $\operatorname{Spin}(7)$ and $\operatorname{Spin}(8)$ labels frequently. In the infra-red, the dual scalar $\phi^{8}$ combines with the seven scalars $\phi^{i}$ to give a vector $\phi^{m}$ of $\operatorname{Spin}(8)$ where $m=1, \ldots, 8$. The fermions lift to the $\left(\mathbf{2}, \mathbf{8}_{\mathbf{c}}\right)$ representation of $S O(2,1) \times \operatorname{Spin}(8)$. The supersymmetry

\footnotetext{
${ }^{1}$ For non-compact manifolds like the monopole moduli spaces, the integral of the Euler density is actually weakly metric dependent rather than being simply topological. For this reason, what is usually called the Euler index need not be integer. In the case of D0-branes, as an analogous example, the bulk contribution to the $L^{2}$ index is actually not integer.
} 
transformations then take the form,

$$
\begin{aligned}
\delta \phi^{m} & =-i \epsilon_{\dot{b}}^{\alpha} \gamma_{\dot{b} a}^{m} \psi_{\alpha a} \\
\delta \psi_{\alpha a} & =\epsilon_{\dot{b}}^{\beta} \gamma_{\dot{b} a}^{m} \tau_{\beta \alpha}^{\mu} \partial_{\mu} \phi^{m},
\end{aligned}
$$

where we have introduced a Grassmann parameter $\epsilon_{\dot{a}}^{\alpha}$ transforming in the $\left(\mathbf{2}, \mathbf{8}_{\mathbf{s}}\right)$ of the symmetry group. An explicit realization for the $\tau^{\mu}$ where $\mu=0,1,2$ can be given in terms of the Pauli matrices,

$$
\tau^{0}=-i \sigma_{2}, \quad \tau^{1}=\sigma_{3}, \quad \tau^{2}=\sigma_{1} .
$$

With spacetime signature $(-,+,+)$, the $\tau^{\mu}$ satisfy the relations:

$$
\begin{aligned}
\left\{\tau^{\mu}, \tau^{\nu}\right\} & =2 \eta^{\mu \nu} \\
{\left[\tau^{\mu}, \tau^{\nu}\right] } & =2 \epsilon^{\mu \nu \lambda} \tau_{\lambda} .
\end{aligned}
$$

We will actually only need to know the variation of the bosons $\phi^{m}$. When the $F^{4}$ terms are included, there are higher derivative corrections to both $\delta \phi$ and $\delta \psi$ in 2.1 but as in [9], they will not play a role in determining the four derivative terms.

As in the case of lower dimensional theories, we will focus on the eight fermion terms in the supersymmetric completion of the $F^{4}$ term. If we schematically denote the eight fermion terms by,

$$
f^{(8)}(\phi) \psi^{8}
$$

then it is easy to see that the variation of 2.3 in the Lagrangian using 2.1 contains a piece with seven fermions and a piece with nine fermions. The same argument given in [9] allows us to conclude that the variation of no other term in the Lagrangian contains nine fermions. Therefore, the piece with nine fermions in the variation of 2.3 must vanish giving the equation,

$$
\gamma_{\dot{b} a}^{m} \psi_{\alpha a} \frac{\partial}{\partial \phi^{m}}\left(f^{(8)}(\phi) \psi^{8}\right)=0
$$

for every $\dot{b}$ and $\alpha$. To proceed, we need to know something about the possible eight fermion terms.

\subsection{The basic fermion bilinears}

The eight fermion terms contain no spacetime derivatives and must be invariant under the Lorentz group $S O(2,1)$. They can be built from a few 
fundamental forms bilinear in the fermions. To count the number of basic bilinears, we note that

$$
[(\mathbf{2}, \mathbf{8}) \otimes(\mathbf{2}, \mathbf{8})]_{a}=(\mathbf{3}, \mathbf{7}) \oplus(\mathbf{3}, \mathbf{2 1}) \oplus(\mathbf{1}, \mathbf{1}) \oplus(\mathbf{1}, \mathbf{3 5}),
$$

as representations of $S O(2,1) \times \operatorname{Spin}(7)$. If we let $\gamma^{i}$ be a basis for the $\operatorname{Spin}(7)$ Clifford algebra then we can define, ${ }^{1}$

$$
\begin{aligned}
\gamma^{i j} & =\frac{1}{2 !}\left(\gamma^{i} \gamma^{j}-\gamma^{j} \gamma^{i}\right) \\
\gamma^{i j k} & =\frac{1}{3 !}\left(\gamma^{i} \gamma^{j} \gamma^{k}-\gamma^{j} \gamma^{i} \gamma^{k}+\ldots\right) .
\end{aligned}
$$

The antisymmetric matrices $\left\{\gamma^{i}, \gamma^{i j}\right\}$ and the symmetric matrices $\left\{1, \gamma^{i j k}\right\}$ constitute a basis out of which we can construct all operators involving fermions. The four basic bilinears appearing in 2.5 are easily found,

$$
\bar{\psi} \tau^{\mu} \gamma^{i} \psi, \quad \bar{\psi} \tau^{\mu} \gamma^{i j} \psi, \quad \bar{\psi} \psi, \quad \bar{\psi} \gamma^{i j k} \psi
$$

where $\bar{\psi}=\psi^{T} \tau_{0}$. Using various Fierz identities, we could in principle determine all possible eight fermion structures. Instead, we will take a different route to fix all possible eight fermion terms. The only observation we need to make concerns the spacetime indices. To get a Lorentz invariant structure, we will eventually need to contract all $\mu$ indices. However, because of the following Fierz identity,

$$
\left(\tau^{\mu}\right)_{\alpha \beta}\left(\tau_{\mu}\right)_{\sigma \delta}=\delta_{\alpha \beta} \delta_{\sigma \delta}-2 \delta_{\alpha \delta} \delta_{\sigma \beta}
$$

we actually only need to consider the last two structures in 2.7. This allows us to observe that any pure fermion structure must contain equal numbers of $\psi_{1 a}$ and $\psi_{2 a}$.

There is a second way to see this condition. Let $T$ denote a diagonal element of the Lorentz group $S L(2, \mathbb{R})$ with eigenvalues $t>1$ and $t^{-1}$. Then we may pick a basis such that,

$$
T \psi_{1 a}=t \psi_{1 a}
$$

and

$$
T \psi_{2 a}=t^{-1} \psi_{2 a}
$$

Clearly then an eight fermion term which is invariant under $T$ must have four $\psi_{1 a}$ and four $\psi_{2 a}$ fermions. We can now turn to the general form of the eight fermion terms.

\footnotetext{
${ }^{1}$ When we want to view these $\operatorname{Spin}(7)$ gamma matrices as part of the $\mathbf{8}_{\mathbf{c}}$ or $\mathbf{8}_{\mathbf{s}}$ representations of $\operatorname{Spin}(8)$, we will use dotted indices; for example, $\gamma_{\dot{a} b}$.
} 


\subsection{Classifying the possible eight fermion terms}

First note that the eight fermion structures that can be constructed from the last two basic bilinears 2.7 can be contracted with at most four scalar fields. Further $\phi^{8}$ can only appear in the form $e^{i n \phi^{8} / g^{2}}$ since there are no spacetime derivatives. We will normalize $\phi^{8}$ to have period $2 \pi g^{2}$. We can then classify eight fermion terms in the following way,

$$
T_{m_{0}}, \quad \phi^{i} T_{m_{1}}^{i}, \quad \phi^{i} \phi^{j} T_{m_{2}}^{i j}, \quad \phi^{i} \phi^{j} \phi^{k} T_{m_{3}}^{i j k}, \quad \phi^{i} \phi^{j} \phi^{k} \phi^{l} T_{m_{4}}^{i j k l},
$$

where $T$ is an eight fermion structure and $i, j, k, l<8$. The label $m_{k}=$ $1, \ldots, N_{k}$ and $N_{k}$ is the number of independent structures that contain $k$ scalars. For example, the possible eight fermion terms with four scalars can take the form,

$$
\sum_{m=1}^{N_{4}} f_{n}^{4, m}(r) e^{i n \phi^{8} / g^{2}}\left(\phi^{i} \phi^{j} \phi^{k} \phi^{l} T_{m}^{i j k l}\right),
$$

under the assumption that there are any such four scalar structures. Here we have defined $r$ to be $\sqrt{\phi^{i} \phi^{i}}$. Now as in $[9,10]$, we can obtain a weaker condition than 2.4 by applying the operator,

$$
\gamma_{c \dot{b}}^{n} \frac{d}{d \psi_{\alpha c}} \partial_{\phi^{n}}
$$

to 2.4. After summing over $\dot{b}$, we obtain the condition:

$$
\Delta\left(f^{(8)}(\phi) \psi^{8}\right)=0
$$

where the Laplacian $\Delta$ includes $\phi^{8}$.

Let us start by considering the restrictions imposed by the weaker constraint 2.9. First note that the Laplacian commutes with the Fourier expansion so we obtain an equation on each Fourier coefficient. Also note that 2.9 does not mix structures with an odd number of scalars and structures with an even number of scalars. This is not true of the stronger condition 2.4. Let us start by writing the first of three coupled differential equations from 2.9 for the even scalar structures:

$$
\left(\frac{d^{2}}{d r^{2}}+\frac{14}{r} \frac{d}{d r}-\frac{n^{2}}{g^{4}}\right) f_{n}^{4, m}=0 .
$$

This gives a standard differential equation for each $m$ and $n$. The perturbative contribution corresponds to $n=0$ and the general solution is,

$$
f_{0}^{4, m}=\frac{c_{0}}{r^{13}}+d_{0}
$$


We can set $d_{0}=0$ since we demand a solution that decays as $r \rightarrow \infty$. The remaining solution is then precisely the one-loop contribution and corresponds to a $\frac{1}{r^{5}} F^{4}$ term. The coefficient $c_{0}$ is then fixed by a one-loop computation. We can conclude that if there are any non-vanishing four scalar structures then they receive only a one-loop contribution. Before analyzing the nonperturbative terms with $n \neq 0$, let us consider the remaining equations.

The second equation mixes the two scalar and four scalar structures:

$$
\begin{gathered}
\sum_{p=1}^{N_{2}}\left(\frac{d^{2}}{d r^{2}}+\frac{10}{r} \frac{d}{d r}-\frac{n^{2}}{g^{4}}\right) f_{n}^{2, p} e^{i n \phi^{8} / g^{2}}\left(\phi^{i} \phi^{j} T_{p}^{i j}\right)= \\
-12 \sum_{m=1}^{N_{4}} f_{n}^{4, m} e^{i n \phi^{8} / g^{2}}\left(\phi^{i} \phi^{j} \delta_{k l} T_{m}^{i j k l}\right) .
\end{gathered}
$$

Again we will first consider the perturbative contributions with $n=0$. There are two possible scenarios: either there is a $\sim 1 / r^{13}$ source term for the differential equation for a two scalar structure labelled by $p$, or there is no source term. In the latter case, the solution takes the form

$$
f_{0}^{2, p} \sim \frac{1}{r^{9}}
$$

but this corresponds to a negative power of the coupling. All such solutions are unphysical. The only possible physical (perturbative) two scalar structures must appear with source terms. They therefore descend naturally from four scalar structures and always have the form,

$$
\phi^{i} \phi^{j} \delta_{k l} T_{m}^{i j k l},
$$

for some four scalar structure. In this case, we find that

$$
f_{0}^{2, p}=-\frac{6}{11} \frac{c_{0}}{r^{11}}
$$

which is again one-loop exact.

The last equation for the even structures mixes the no scalar and two scalar structures:

$$
\sum_{q=1}^{N_{2}}\left(\frac{d^{2}}{d r^{2}}+\frac{6}{r} \frac{d}{d r}-\frac{n^{2}}{g^{4}}\right) f_{n}^{0, q} e^{i n \phi^{8} / g^{2}} T_{q}=-2 \sum_{p=1}^{N_{2}} f_{n}^{2, p} e^{i n \phi^{8} / g^{2}}\left(\delta_{i j} T_{p}^{i j}\right) .
$$

Without a source term, we find that

$$
f_{0}^{0, q} \sim \frac{1}{r^{5}}
$$


which requires negative powers of the coupling. We therefore need to consider only zero scalar structures that descend from four scalar structures, and we find that

$$
f_{0}^{0, q}=\frac{1}{33} \frac{c_{0}}{r^{9}}
$$

which is again one-loop exact. The even structures are therefore completely determined at the perturbative level by classifying the four fermion structures.

Let us turn to the odd scalar structures. The first equation constrains the three scalar structures alone:

$$
\left(\frac{d^{2}}{d r^{2}}+\frac{12}{r} \frac{d}{d r}-\frac{n^{2}}{g^{4}}\right) f_{n}^{3, m}=0
$$

When $n=0$, the solution takes the form

$$
f_{0}^{3, m} \sim \frac{1}{r^{11}}
$$

which requires no power of the coupling. Since the structure cannot appear at tree-level, there are no perturbative three scalar structures. The final equation relates the three and one scalar structures:

$$
\begin{aligned}
& \sum_{p=1}^{N_{1}}\left(\frac{d^{2}}{d r^{2}}+\frac{8}{r} \frac{d}{d r}-\frac{n^{2}}{g^{4}}\right)\left(f_{n}^{1, p}\right) e^{i n \phi^{8} / g^{2}}\left(\phi^{i} T_{p}^{i}\right) \\
& =-6 \sum_{m=1}^{N_{3}} f_{n}^{3, m} e^{i n \phi^{8} / g^{2}}\left(\phi^{i} \delta_{j k} T_{m}^{i j k}\right) .
\end{aligned}
$$

When $n=0$, the solution is of the form

$$
f_{0}^{1, p} \sim \frac{1}{r^{7}}
$$

which requires a negative power of the coupling constant. Therefore, there are no possible odd scalar structures at the perturbative level. ${ }^{1}$ Since we know there is a non-vanishing one-loop contribution to the $F^{4}$ terms, we can also conclude that there must be at least one possible four scalar structure.

\footnotetext{
${ }^{1}$ We wish to thank N. Seiberg for pointing out the following simple argument that excludes odd scalar structures at the perturbative level. The Weyl group action given in (3.2) inverts all the scalar fields including $\phi^{8}$. Since $\phi^{8}$ does not appear perturbatively, this symmetry excludes any odd scalar structure.
} 


\subsection{Non-perturbative solutions}

At the perturbative level, we have demonstrated that the $F^{4}$ terms receive only one-loop contributions just by using the weak constraint 2.9. We can now consider the non-perturbative solutions where $n \neq 0$. Consider the equation,

$$
\left(\frac{d^{2}}{d r^{2}}+\frac{2 \nu+1}{r} \frac{d}{d r}-\frac{n^{2}}{g^{4}}\right) f_{n}(r)=0
$$

It is convenient to replace $f_{n}(r)$ by $r^{\nu} f_{n}(r)$, which takes the equation to the form:

$$
\left(\frac{d^{2}}{d r^{2}}+\frac{1}{r} \frac{d}{d r}-\frac{n^{2}}{g^{4}}-\frac{\nu^{2}}{r^{2}}\right) r^{\nu} f_{n}(r)=0 .
$$

The solution to this equation is given in terms of $K_{\nu}(z)$, a modified Bessel function of the third kind:

$$
f_{n}(r)=c_{n} n^{1 / 2} \frac{1}{g^{24}}\left(\frac{g^{2}}{r}\right)^{\nu} K_{\nu}\left(n r / g^{2}\right)
$$

Here $c_{n}$ is a constant yet to be determined and we have inserted powers of the coupling needed on dimensional grounds. Most of the time, $\nu$ will be half-integer and then $K_{\nu}(z)$ has the following useful expansion [16],

$$
K_{n+1 / 2}(z)=(\pi / 2 z)^{1 / 2} e^{-z} \sum_{m=0}^{n}(2 z)^{-m} \frac{\Gamma(n+m+1)}{m ! \Gamma(n-m+1)} .
$$

Before solving the differential equations that we found in the previous section, some dimensional analysis is in order. It is convenient to rescale the coupling into the scalar fields and fermions. After this rescaling the fields $\phi$ have dimension $1 / 2$ while the fermions have dimension 1 . The kinetic term takes the form,

$$
\int d^{3} x \frac{1}{2} \partial_{\mu} \phi \partial^{\mu} \phi
$$

with no explicit powers of the coupling and with $\phi^{8}$ included. The one-loop corrected $F^{4}$ terms take the form,

$$
\frac{1}{g} \int d^{3} x \frac{1}{r^{5}}\left(\partial_{\mu} \phi^{i} \partial^{\mu} \phi^{i}\right)^{2}
$$

with a single power of $1 / g$. The powers of $g$ are simply fixed on dimensional grounds since the action is dimensionless. The one-loop corrected effective 
action is not scale-invariant. In the limit of low-energies where $g \rightarrow \infty$, the theory must become scale-invariant. All explicit powers of $g$ must disappear from the action. We will see how the instanton corrections to the $F^{4}$ term combine with the one-loop correction to give a scale and Spin(8) invariant result in the strong coupling limit as suggested in [8].

Let us turn to the solution for the four scalar structure equation 2.10. In this case, $\nu=13 / 2$ and the solution for the $n$ instanton sector is given by,

$$
\begin{aligned}
f_{n}^{4, m} e^{i n \phi^{8} / g} & =c_{n} n^{1 / 2} \frac{1}{g^{14}}\left(\frac{g}{r}\right)^{13 / 2} K_{13 / 2}(n r / g) e^{i n \phi^{8} / g} \\
& =c_{n} n^{1 / 2} \frac{1}{g^{14}}\left(\frac{\pi}{2 n}\right)^{1 / 2} e^{-\frac{|n| r}{g}+\frac{i n \phi^{8}}{g}}\left(\frac{g^{7}}{r^{7}}+\ldots\right),
\end{aligned}
$$

which is exactly the form that we would expect for an $n$ instanton sector contribution.

When the four scalars are taken into account, we see that the norm of the four scalar term goes like $\sim r^{-3} e^{-|n| r / g}$ up to lower order terms. A similar analysis shows that the $k$ scalar term can be written as a sum of a solution to the homogeneous Laplace equation with leading order behavior $\sim r^{-3} e^{-|n| r / g}$ and a solution to the inhomogeneous equation with leading order behavior $\sim r^{-4} e^{-|n| r}$. Set

$$
\gamma^{r}=\frac{1}{r} \phi^{j} \gamma^{j}
$$

Isolating the part of the constraint equation 2.4 which equates terms of order $r^{-3} e^{-|n| r / g}$ gives for nonzero $n$,

$$
\left(\gamma_{\dot{a} b}^{r}-i \operatorname{sign}(\mathrm{n}) \gamma_{\dot{a} b}^{8}\right) \psi_{\alpha b} h=0,
$$

where $h$ denotes the $O\left(r^{-3} e^{-|n| r}\right)$ eight fermion terms. This equality comes from differentiating only the exponential $e^{-r|n| / g+i n \phi^{8} / g}$ in the constraint equation. Once again we pass to a weaker but more pliable equation. We apply the operator $\gamma_{c \dot{a}}^{r} \partial_{\psi_{\alpha c}}$ to the above inequality, and sum over $\dot{a}$ and $\alpha$ to obtain:

$$
\partial_{\psi_{\alpha b}}\left(\psi_{\alpha b} h\right)+i \operatorname{sign}(\mathrm{n}) \gamma_{c b}^{r 8} \psi_{\alpha b} \partial_{\psi_{\alpha c}} h=0
$$

Using,

$$
\partial_{\psi_{\alpha t}}\left(\psi_{\alpha t} h\right)=8 h
$$

we find that,

$$
\left(8-i \operatorname{sign}(\mathrm{n}) \gamma_{s t}^{r 8} \psi_{\alpha s} \partial_{\psi_{\alpha t}}\right) h=0
$$


We have already shown that any eight fermion term contains an equal number of $\psi_{1 a}$ and $\psi_{2 a}$. We can therefore build $h$ from terms of the form,

$$
\psi_{1} \gamma^{m_{1} p_{1}} \psi_{1} \psi_{1} \gamma^{m_{2} p_{2}} \psi_{1} \psi_{2} \gamma^{m_{3} p_{3}} \psi_{2} \psi_{2} \gamma^{m_{4} p_{4}} \psi_{2}
$$

where $1 \leq m_{i}, p_{i} \leq 8$. By including $\gamma^{8}$ in $\gamma^{m p}$, we automatically include both antisymmetric basis elements $\left\{\gamma^{i}, \gamma^{i j}\right\}$.

The operator $i \operatorname{sign}(\mathrm{n}) \gamma_{b c}^{r 8} \psi_{\alpha b} \partial_{\psi_{\alpha c}}$ acts on a bilinear of the form $\psi_{\beta} \gamma^{m p} \psi_{\beta}$ by sending it to,

$$
\psi_{\beta} i \operatorname{sign}(\mathrm{n})\left[\gamma^{r 8}, \gamma^{m p}\right] \psi_{\beta} .
$$

An easy computation gives eigenvalues of $2,0,-2$ for this operation on bilinears. The largest eigenvalue is associated to eigenvectors of the form,

$$
\psi_{\beta}\left(\gamma^{p r} \pm i \operatorname{sign}(\mathrm{n}) \gamma^{p 8}\right) \psi_{\beta},
$$

where $p \neq r, 8$. Then for $n$ positive, the only possible way to obtain an eigenvalue of 8 for the eight fermion term as required by 2.24 is if the eight fermion term is of the form, ${ }^{1}$

$$
\begin{aligned}
& a_{1}\left[\psi_{1}\left(\gamma^{p r}-i \gamma^{p 8}\right) \psi_{1} \psi_{1}\left(\gamma^{p r}-i \gamma^{p 8}\right) \psi_{1} \psi_{2}\left(\gamma^{q r}-i \gamma^{q 8}\right) \psi_{2} \psi_{2}\left(\gamma^{q r}-i \gamma^{q 8}\right) \psi_{2}\right] \\
+ & a_{2}\left[\psi_{1}\left(\gamma^{p r}-i \gamma^{p 8}\right) \psi_{1} \psi_{1}\left(\gamma^{q r}-i \gamma^{q 8}\right) \psi_{1} \psi_{2}\left(\gamma^{p r}-i \gamma^{p 8}\right)\right. \\
\times & \left.\psi_{2} \psi_{2}\left(\gamma^{q r}-i \gamma^{q 8}\right) \psi_{2}\right]
\end{aligned}
$$

for constants $a_{1}$ and $a_{2}$. Let us use $s\left(a_{1}, a_{2}\right)$ to denote the structure in 2.25 for convenience. In fact, up to a scalar factor, there is a unique combination of $a_{1}$ and $a_{2}$ allowed. This can be seen in the following way: equation 2.23 implies that,

$$
\left(I-i \gamma^{r 8}\right)_{a s} \psi_{\alpha s} h=0 .
$$

Note that $\left(I-i \gamma^{r 8}\right) / 2$ is a Hermitian projection operator with the complementary orthogonal projection given by its conjugate. Therefore, we see that it has four zero and four non-zero eigenvalues on each chiral subspace since conjugation preserves chirality. Now for an eight fermion term to be annihilated by each of the eight non-zero fermions $\left(I-i \gamma^{r 8}\right)_{a s} \psi_{\alpha s}$ simply means that it can be expressed as a product of these eight fermions. This uniquely fixes the ratio of $a_{1}$ and $a_{2}$ (unless the two structures are the same). Observe that in this notation, the scalar structures are mixed. The scalars are hidden in the definition of $\gamma^{r}$. The entire eight fermion term for the Fourier mode $n \neq 0$ is then determined by the four scalar solution 2.22 .

\footnotetext{
${ }^{1}$ For $n$ negative, we replace each bilinear $\psi\left(\gamma^{m r}-i \gamma^{m 8}\right) \psi$ by $\psi\left(\gamma^{m r}+i \gamma^{m 8}\right) \psi$.
} 
Therefore the eight fermion term is uniquely fixed (up to constants). In the sector with instanton number $n$ positive, $h$ takes the form:

$$
c_{n} n^{1 / 2} \frac{1}{g^{14}}\left(\frac{g}{r}\right)^{13 / 2} r^{4} e^{-n r / g}\left(\frac{\pi g}{2 n r}\right)^{1 / 2} e^{i n \phi^{8} / g} s\left(a_{1}, a_{2}\right)
$$

Therefore, the solution of the homogeneous Laplace equation for the four scalar term is given by,

$$
\begin{gathered}
c_{n} n^{1 / 2} \frac{1}{g^{14}}\left(\frac{g}{r}\right)^{13 / 2} r^{4} K_{13 / 2}(n r / g) e^{i n \phi^{8} / g} \\
{\left[a_{1} \psi_{1} \gamma^{p r} \psi_{1} \psi_{1} \gamma^{p r} \psi_{1} \psi_{2} \gamma^{q r} \psi_{2} \psi_{2} \gamma^{q r} \psi_{2}\right.} \\
\left.+a_{2} \psi_{1} \gamma^{p r} \psi_{1} \psi_{1} \gamma^{q r} \psi_{1} \psi_{2} \gamma^{p r} \psi_{2} \psi_{2} \gamma^{q r} \psi_{2}\right]
\end{gathered}
$$

Similarly the coefficients of the different scalar structures in the solution of the homogeneous Laplace equation can simply be extracted from 2.26 by taking the $k$ scalar component of 2.26 and replacing $e^{-n r / g}(\pi g / 2 n r)^{1 / 2}$ by the appropriate Bessel function, $K_{5 / 2+k}(r n / g)$. These then determine the solutions to the inhomogeneous equations. In this way, the eight fermion term is completely determined in the sector with instanton number $n$. What remains is for us to determine the coefficients $c_{n}$.

\section{$3 \quad$ Scale and Spin(8) Invariance}

\subsection{Constraints from gauge invariance}

In the strong coupling limit, the effective action must become scale and Spin(8) invariant. The eight fermion term 2.26 contains both even and odd structures. Both are needed for $\operatorname{Spin}(8)$ invariance. Schematically, the three scalar structure goes over to the term,

$$
\sum_{n} f_{n}^{3}(r) \phi^{i} \phi^{j} \phi^{k} e^{i n \phi^{8} / g} T^{i j k} \rightarrow \phi^{i} \phi^{j} \phi^{k} \phi^{8} T^{i j k 8},
$$

in the strong coupling limit, and similarly for the one scalar term. However, we will not actually need to assume $\operatorname{Spin}(8)$ invariance to determine the constants $c_{n}$. First note that hermiticity of the eight fermion term implies that,

$$
c_{-n}=c_{n}^{*}
$$


Since we are considering the effective action of a full $S U(2)$ Yang-Mills theory, we also need to impose invariance under the residual Weyl group action:

$$
\phi^{i} \rightarrow-\phi^{i}, \quad \phi^{8} \rightarrow-\phi^{8}, \quad \psi \rightarrow-\psi .
$$

Invariance under the Weyl group then implies that,

$$
c_{-n}=c_{n},
$$

so the coefficients $c_{n}$ are real.

\subsection{Determining the $c_{n}$ coefficients}

Let us now show that the $g \rightarrow \infty$ limit results in a $\operatorname{Spin}(8)$ invariant eight fermion term. Alternately, the $c_{n}$ coefficients can be determined by assuming the $\operatorname{Spin}(8)$ invariance of the strong coupling limit. We will see that our argument gives results which agree with this second approach, and hence constitutes an independent argument for Spin(8) invariance.

So far, we have shown that the perturbative eight fermion term is oneloop exact with the coefficient $c_{0}$ fixed by the loop computation. The perturbative eight fermion term is completely determined by specifying the four scalar structure. We have also shown that the instanton sector $n$ eight fermion term is completely determined up to a constant $c_{n}$. Let us focus on the $g$ dependence of the eight fermion term, which we will denote $f_{g}$ where $f_{g}=f_{n}(g) e^{i n \phi^{8} / g}$. The full Yang-Mills theory is well-behaved at low-energies since away from the singularities in the moduli space, the theory is infra-red free. We can therefore assume that the limit of the eight fermion term $f_{g}$ and its first six derivatives exists as $g \rightarrow \infty$ and converges to a limit $F$ and its first six derivatives in the following sense: the eight fermion term $F$ which describes the strong coupling limit can be made periodic with period $2 \pi g$ by averaging,

$$
F_{g}=\sum_{j \in \mathbb{Z}} F\left(\phi^{8}+2 \pi g j\right)
$$

We can express $F_{g}$ in terms of its Fourier modes $\hat{F}(n / 2 \pi g)$,

$$
F_{g}=\sum_{n \in \mathbb{Z}} \frac{1}{2 \pi g} \hat{F}(n / 2 \pi g) e^{i n \phi^{8} / g} .
$$

Note that we are not assuming that $F$ is $\operatorname{Spin}(8)$ invariant. The appropriate notion of convergence of $f_{g}$ to $F$ is that,

$$
g^{k}\left[f_{n}(g)-\frac{1}{2 \pi g} \hat{F}(n / 2 \pi g)\right]
$$


tends to zero as $g \rightarrow \infty$ for $0 \leq k \leq 6$. In fact, if this were not the case, the notion of an effective action would make little sense. We will see why we need the first six derivatives momentarily.

On dimensional grounds, we know that $f_{n}=c_{n} \frac{1}{g^{7}} h_{n}$ where $h_{n}$ is determined from 2.26 by choosing $h_{n}$ to be normalized so that $n$ and $g$ only appear together in the combination $|n| / g$. Now the same analysis we applied to $f_{g}$ must apply equally to $F_{g}$ since it satisfies the same constraint equations. So there is an expansion,

$$
\frac{1}{2 \pi} \hat{F}(n / 2 \pi g)=C_{n} h_{n}
$$

The left-hand-side depends on $n$ and $g$ only in the combination $n / g$ and therefore the same is true for the right-hand-side. Then $C_{n}=C(n / g)$ and,

$$
c_{n}-g^{6} C(n / g) \rightarrow 0 \text {. }
$$

We therefore learn that the first six terms in a Taylor expansion of $C(n / g)$ about zero vanish, which implies that

$$
c_{n}-g^{6} C^{(6)}(0) \frac{1}{6 !}\left(\frac{n}{g}\right)^{6} \rightarrow 0
$$

as $g$ tends to infinity. Finally, we can conclude that for $n>0$ :

$$
c_{n}=c \frac{n^{6}}{6 !} .
$$

Note that this formula does not imply that $c_{0}=0$ because $h_{n}$ is singular at $n=0$. Since we have determined the strong coupling limit of the eight fermion term by determining $c_{n}$, we can Fourier expand the limiting form and extract the coefficient $c_{0}$. Therefore, the constant $c$ in 3.7 is fixed in terms of $c_{0}$. For our subsequent discussion, we will not need to determine this constant explicitly.

\subsection{A comparision with the $\operatorname{Spin}(8)$ result}

We will show by direct computation that 3.7 gives an eight fermion term which converges to a Spin(8) invariant term in the strong coupling limit. More accurately, we will compute the Fourier coefficients of the unique $\operatorname{Spin}(8)$ invariant term and find agreement with 3.7. 
We now set $F$ to be the following harmonic four scalar eight fermion term

$$
\begin{array}{ll}
\sum_{i, j, m, l \leq 8} & \frac{1}{\left(r^{2}+\left(\phi^{8}\right)^{2}\right)^{7}} \phi^{i} \phi^{j} \phi^{m} \phi^{l} \\
& \times\left[a_{1} \psi_{1} \gamma^{p i} \psi_{1} \psi_{1} \gamma^{p j} \psi_{1} \psi_{2} \gamma^{q m} \psi_{2} \psi_{2} \gamma^{q l} \psi_{2}\right. \\
& \left.+a_{2} \psi_{1} \gamma^{p i} \psi_{1} \psi_{1} \gamma^{q j} \psi_{1} \psi_{2} \gamma^{p m} \psi_{2} \psi_{2} \gamma^{q l} \psi_{2}\right]
\end{array}
$$

The form of this term is simply fixed on dimensional grounds since the effective action must be scale-invariant.

We can extract the $c_{n}$ coefficients from the four scalar part of 3.8 where none of the four scalars is $\phi^{8}$. We then need to compute the Fourier transform in the $\phi^{8}$ direction of,

$$
\frac{1}{\left(r^{2}+\left(\phi^{8}\right)^{2}\right)^{7}}
$$

The Fourier component $\hat{F}(n / g)$ is given by,

$$
\hat{F}(n / g)=\frac{2}{6 !}\left(\frac{n}{2 g r}\right)^{13 / 2} \pi^{1 / 2} K_{13 / 2}(r n / g) .
$$

We can now see that the homogeneous four scalar term in $F_{g}$ is given by,

$$
\begin{aligned}
& \frac{1}{\pi g} \sum_{n \in \mathbb{Z}} \frac{\pi^{1 / 2}}{6 !} K_{13 / 2}(r n / g) e^{i n \phi^{8} / g}\left(\frac{n}{2 g r}\right)^{13 / 2} r^{4} \\
& \times\left[a_{1} \psi_{1} \gamma^{p r} \psi_{1} \psi_{1} \gamma^{p r} \psi_{1} \psi_{2} \gamma^{q r} \psi_{2} \psi_{2} \gamma^{q r} \psi_{2}\right. \\
& \left.+a_{2} \psi_{1} \gamma^{p r} \psi_{1} \psi_{1} \gamma^{q r} \psi_{1} \psi_{2} \gamma^{p r} \psi_{2} \psi_{2} \gamma^{q r} \psi_{2}\right]
\end{aligned}
$$

The coefficient $C_{n}$ extracted from 3.10 agrees with the formula 3.7 .

\section{A Comparision With Semi-Classical Computa- tions}

The leading terms in the expansion of 2.26 should match semi-classical instanton computations performed in $[5,6]$. For example, the leading contribution to the zero scalar structure in 2.26 takes the form,

$$
\frac{1}{r^{3}} e^{-r+i \phi^{8}}
$$


in the one-instanton sector. This agrees with the term in the effective action computed in [5] up to normalization choices.

For higher instanton number, the situation is much more interesting. Let us recall that instantons in three-dimensional gauge theory correspond to monopole field configurations in four-dimensional gauge theory. Unlike the case of four-dimensional gauge theory, instanton computations in three dimensions are naturally performed in the broken gauge theory. Monopole solutions have a scale set by the choice of vacuum expectation value so integrals over monopole moduli space do not require the use of cut-off techniques like constrained instantons used in four dimensions.

This simple observation implies a quite beautiful relation between the geometry of monopole moduli space and the terms in the effective action that we have determined. Let us recall that $n$ monopole moduli space $\mathcal{M}_{n}$ is a $4 n$-dimensional hyperKähler manifold which has a standard isometric decomposition,

$$
\mathcal{M}_{n}=\mathbb{R}^{3} \times \frac{S^{1} \times \mathcal{M}_{n}^{0}}{\mathbb{Z}_{n}}
$$

The $\mathbb{R}^{3} \times S^{1}$ parametrize the center of mass and the coordinate conjugate to the electric charge. Quantization of these moduli in four-dimensional $N=4$ Yang-Mills leads to a supersymmetric sigma model on $\mathcal{M}_{n}$ described in [17]. Note that it follows from [18] that there are $8 n$ real fermionic zero modes in a charge $n$ monopole background. These zero modes become superpartners to the bosonic moduli in the supersymmetric sigma model.

At first sight, it might seem that the expectation value of an eight fermion term in the $S U(2)$ Yang-Mills theory would vanish in a sector with instanton number $|n|>1$ because of fermion zero mode counting. However, this is not the case because the moduli for the relative motion of the monopoles do not parametrize a flat space. Rather the semi-classical instanton contribution is proportional to the high temperature limit of the twisted partition function on monopole moduli space,

$$
\chi_{n}=\int_{\mathcal{M}_{n}^{0}} \lim _{\beta \rightarrow 0} \operatorname{tr}(-1)^{F} e^{-\beta H}(x, x)
$$

The Hamiltonian is the one describing supersymmetric quantum mechanics on $\mathcal{M}_{n}$ [17]. This is the bulk contribution to the $L^{2}$ index which we would want to compute in sectors with a given electric charge to prove, for example, the conjecture by Sen about dyon bound states [19]. The high temperature limit is a perturbative limit of the partition function and the term $\chi_{n}$ reduces 
to the integral of the Euler density over $\mathcal{M}_{n}^{0}$,

$$
\chi_{n}=\int_{\mathcal{M}_{n}^{0}} e\left(T \mathcal{M}_{n}^{0}\right)
$$

where $e\left(T \mathcal{M}_{n}^{0}\right)$ is the Euler class. If the relative monopole moduli space $\mathcal{M}_{n}^{0}$ had been compact, $\chi_{n}$ would be the Euler index. For $n=2$, the Chern-Gauss-Bonnet theorem can be used to show that $\chi_{2}$ is the topological Euler characteristic $\chi_{n}^{t o p}$. This seems likely to remain true for all $n$, but a careful proof of this equality for $n>2$ requires a better understanding of the monopole moduli space metric.

Since we have determined the exact eight fermion terms from supersymmetry, we should be able to extract $\chi_{n}$ from our solution by matching the leading semi-classical behavior. This seems quite remarkable.

Fortunately for us, there has been a great deal of interesting work on semi-classical instanton computations in [6,20]. From the analysis of the measure for the $n$ instanton computation performed in [6], we can conclude that:

$$
\frac{c_{n}}{c_{1}}=n^{5} \chi_{n}
$$

However, from 3.7 we see that

$$
\frac{c_{n}}{c_{1}}=n^{6}
$$

and therefore $\chi_{n}=n$. Remarkably, the results obtained in [21] have been used in [6] to show that $\chi_{n}^{t o p}$ is also $n$. Clearly, a deeper explanation of why supersymmetry should determine $\chi_{n}$ is needed.

\section{Acknowledgements}

It is our pleasure to thank W. Fischler, D. Kabat and especially N. Seiberg for helpful conversations. The work of S.P. is supported by NSF grant PHY-9511632, that of S.S. by NSF grant DMS-9627351 and that of M.S. by NSF grant DMS-9505040 and NSF grant DMS-9870161. 


\section{References}

[1] S. Sethi and L. Susskind, hep-th/9702101, Phys. Lett. B400 (1997) 265.

[2] T. Banks and N. Seiberg, hep-th/9702187, Nucl. Phys. B497 (1997) 41.

[3] N. Seiberg, hep-th/9705117.

[4] T. Banks, W. Fischler, S. H. Shenker, and L. Susskind, Phys. Rev. D55 (1997) 5112.

[5] J. Polchinski and P. Pouliot, hep-th/9704029, Phys. Rev. D56 (1997) 6601.

[6] N. Dorey, V. Khoze and M. Mattis, hep-th/9704197, Nucl. Phys. B502 (1997) 94.

[7] M. Dine and N. Seiberg, hep-th/9705057, Phys. Lett. B409 (1997) 239.

[8] T. Banks, W. Fischler, N. Seiberg and L. Susskind, hep-th/9705190, Phys. Lett. B408 (1997) 111.

[9] S. Paban, S. Sethi and M. Stern, hep-th/9805018.

[10] S. Paban, S. Sethi and M. Stern, hep-th/9806028.

[11] S. Sethi and M. Stern, hep-th/9705046, Comm. Math. Phys. 194 (1998) 675 .

[12] P. Yi, hep-th/9704098, Nucl. Phys. B505 (1997) 307.

[13] M. B. Green and M. Gutperle, hep-th/9711107, JHEP 01 (1998) 05.

[14] M. B. Green and S. Sethi, hep-th/9808061.

[15] G. Moore, N. Nekrasov and S. Shatashvili, hep-th/9803265.

[16] H. Bateman, ed. A. Erdelyi, Higher Transcendental Functions, vol. 2, McGraw-Hill Book Company, 1953.

[17] J. Blum, hep-th/9401133, Phys. Lett. B333 (1994) 92.

[18] C. Callias, Comm. Math. Phys. 62 (1978) 213;

E. Weinberg, Phys. Rev. D20 (1979) 936.

[19] A. Sen, hep-th/9402002, Int. J. Mod. Phys. A9 (1994) 3707; hepth/9402032, Phys. Lett. B329 (1994) 217. 
[20] N. Dorey, V. Khoze, M. Mattis, D. Tong and S. Vandoren, hepth/9703228, Nucl. Phys. B502 (1997) 59.

[21] G. Segal and A. Selby, Comm. Math. Phys. 177 (1996) 775. 